\title{
Using the Loss-Sensitivity Principle to Explain the Integration of Concurrent Decisions in Computer-based Fictitious Betting Situations
}

\section{Boe $\mathrm{O}^{1 *}$ and Garling $\mathrm{T}^{2}$}

${ }^{1}$ School of Business, University of South-Eastern Norway, Drammen, Norway

${ }^{2}$ Department of Psychology, University of Gothenburg, Haraldsgatan 1, 41314 Goteborg, Sweden

\begin{abstract}
In betting situations, if an outcome of a decision outcome is uncertain, this may counteract a possible integration of concurrent decisions. Integration here means that one add values or utilities to the outcomes that one expects. There has been proposed several possible reasons that could explain why a prior outcome is integrated. The aim of the present study was to investigate whether a proposed loss-sensitivity principle (LSP) could explain the integration of concurrent decisions. According to the LSP, a prior outcome will only be integrated if a decision maker (DM) takes into account a future loss and the disutility of this loss.

Materials and methods: We conducted an experiment in order to test the proposed LSP. 48 undergraduate students participated as subjects in the experiment. The materials consisted of fictitious bets presented on a computer screen. In all the conditions of the experiment, one block consisted of single decisions. Half of these decisions consisted of a choice of either having an even chance of winning a certain amount or nothing and a choice of being sure of winning half the amount. The other half of the decisions consisted of a choice of either having an even chance of losing a certain amount or nothing and a choice of being sure of losing half of the amount.
\end{abstract}

Results: Our results yielded no integration. After a prior loss subjects became more risk-aversive when choosing between losses, partially in accordance with the proposed LSP.

Conclusion: No integration occurred in the conditions with concurrent decisions and prior outcomes.

Keywords: Concurrent decisions; Loss-sensitivity principle; Decision making; Integration; Prior outcome; Uncertainty

\section{Introduction}

It is a well-known fact that it is often challenging to make judgments and decisions. This becomes even more challenging when one is facing several options to choose from. One reason that decision making then becomes challenging is because of uncertainty and value conflicts. In addition, decisions are always separated from each other even if the research literature commonly describes the decisions as being independent from each other [1,2]. Throughout one's daily activities, very often a decision maker (DM) has already made a decision and thus already have incorporated the outcome from a previous choice when the DM has to make a new choice. A DM can also experience difficulties in situations where he or she is required to make several decisions at the same time. These types of situations are referred to as concurrent decisions or concurrent decision making. Within the area of machine learning, a lot of research on concurrent decisions has been conducted. A major part of the research conducted within this area has focused upon decision making under uncertainty based on Markov decision processes (MDPs) for structured actions [3-13]. However, most of these approaches tend to ignore the temporal properties of such problems, and do not address learning and planning with activities that take various amounts of time for completion (e.g., activities modeled as temporally extended actions [14].

Decision making theories are either normative, that is, entailing utility maximization, or they are descriptive. Some of the latter decision making theories explains how people make decisions and they are then similar to normative theories in that they are based on value or utility. Here the decision maker (DM) is assumed to first assign a utility or value $\mathrm{v}(\mathrm{x})$ to each outcome $\mathrm{x}$, and then to select the option with the highest value. An example of a value-based decision making theory is prospect theory developed by Kahneman and Tversky [15], see also Tversky and
Kahneman [16,17]. An alternative "reason-based" approach has been proposed by Shafir et al. [18]. This "reason-based" approach identifies various reasons and arguments that are imported into and influences decision. This approach indicates that choices are explained in terms of the balance of reasons for and against the various options. Lacking a comprehensive descriptive theory, value-based and reason-based analyses are still significant contributors to the understanding of how decisions are made.

Prospect theory is similar to reason-based approaches in its assumption that the DM edits options. This can be seen as a contradiction to the normative decision theory found in economics [19]. Examples of such editing operations include framing outcomes as gains or losses relative to a reference point. Framing of this type also include that a DM segregates or integrates prior outcomes or decisions [20,21]. A definition of integration of the outcomes of two concurrent decisions, suggested by Boe and Gärling [22], is the following: integration is "adding the utilities of the expected outcomes of one decision to the utilities of the expected outcomes of another decision" (p. 114). If a DM uses a "minimal account" as his or her decision frame, this means that the outcomes of a particular choice becomes evaluated independently of prior outcomes. A DM may often use this decision frame because

*Corresponding author: Boe O, School of Business, University of South-Eastern Norway, Drammen, Norway, Tel: +4747023634; E-mail: Ole.Boe@usn.no

Received November 29, 2018; Accepted December 05, 2018; Published December 12, 2018

Citation: Boe O, Garling T (2018) Using the Loss-Sensitivity Principle to Explain the Integration of Concurrent Decisions in Computer-based Fictitious Betting Situations. Arts Social Sci J 9: 423. doi: 10.4172/2151-6200.1000423

Copyright: (c) 2018 Boe O, et al. This is an open-access article distributed under the terms of the Creative Commons Attribution License, which permits unrestricted use, distribution, and reproduction in any medium, provided the original author and source are credited. 
it “..(i) simplifies evaluations and reduces cognitive strain, (ii) reflects the intuition that consequences should be causally linked to acts, and (iii) matches the properties of hedonic experience which is more sensitive to desirable and undesirable changes than to steady states" (p. 457) [21]. In studies by Kahneman and Tversky [15] and Tversky and Kahneman [21] three examples of segregation are provided. One example was the following:

Imagine that you face the following pair of concurrent decisions. First examine both decisions, then indicate the options you prefer.

Decision (1). Choose between:

A. a sure gain of $\$ 240$

B. $25 \%$ chance to gain $\$ 1000$, and $75 \%$ chance to gain nothing

Decision (2). Choose between:

C. a sure loss of $\$ 750$

D. $75 \%$ chance to lose $\$ 1000$, and $25 \%$ chance to lose nothing

The results yielded that a majority of DMs were found to chose options A and D. Contradicting this, when DMs were requested to choose between the following two alternatives they chose option B':

\section{$A^{\prime} .25 \%$ chance to gain $\$ 240$, and $75 \%$ chance to lose $\$ 760$}

$B^{\prime} .25 \%$ chance to gain $\$ 240$, and $75 \%$ chance to lose $\$ 750$

As should be realized, alternative $\mathrm{B}^{\prime}$ is a combination of options $\mathrm{C}$ and $\mathrm{D}$ whereas $\mathrm{A}^{\prime}$ is a combination of options $\mathrm{A}$ and $\mathrm{B}$. A clear picture emerges from this, as the DMs did not seem able to make choices which overall would maximized expected utility. Presumably, they framed each of the decision problems in a minimal account.

It is possible that uncertainty of the outcome of the first decision counteracts integration of concurrent decisions $[23,24]$. Thus, when subjects encounter a prior outcome they may take it into account. Several possible reasons why a prior outcome is integrated have been proposed. Integration of concurrent decisions may be challenging for several reasons. One reason is that the world is stochastic and the uncertain outcomes of executing multiple activities concurrently makes this problem difficult. In regular sequential decision making, an individual would simply select the next action when the current action being executed terminates. However, when concurrent decisions are made, a set of concurrent activities are often executed, and one or more activities may terminate before the rest [25]. Thaler and Johnson [26] argued that subjects integrate when it maximizes utility. Thaler and Johnson [26] further discovered that value becomes maximized when gains are segregated, when losses are integrated, and when small losses are integrated with large gains (known as mixed gains). They also found that the relative size of prior large losses and small gains (mixed losses) was a decisive factor that could explain if value was maximized. On the other hand, Investigating their renewable resources (RR) model, Linville and Fischer [27] found that losses and gains were segregated but mixed gains and mixed losses were integrated. In their study, no restrictions were imposed on whether a loss perceived a gain or the reverse.

Gärling and Romanus [23] proposed a loss-sensitivity hypothesis. According to this hypothesis a prior outcome is only integrated when a DM evaluates the disutility of a future loss, not otherwise. A more encompassing account may in this case be employed because avoiding losses are more important than maximizing gain. In general, the losssensitivity hypothesis predicts risk aversion after a prior loss, risk seeking after a prior gain.
In a previous study by Boe [28] it was investigated whether an attentional bias was capable of explaining why DMs sometimes would fail to integrate the outcomes of concurrent decisions. Support for this attentional bias was found in the sense that participants that expressed a more positive attitude toward driving was found to choose more often to drive to stores that were within walking distance. In comparison, participants that expressed a less positive attitude towards driving were found to choose less often to drive to stores that were within walking distance.

In a further study of concurrent decisions, Boe and Gärling [29] investigated whether outcomes that were certain would be more often integrated than outcomes that were uncertain. They further investigated whether outcomes of concurrent decisions that were causally related would be more often integrated than outcomes that were unrelated. Results from this study revealed that both causally related outcomes and outcomes of concurrent decisions that were uncertain had an effect upon integration.

Continuing this line of thinking, Boe and Gärling [22] conducted an experiment with the purpose of investigating whether options of concurrent decisions that were causally related would not be taken into account and as a result therefore not chosen. This despite that the combinations of options were more attractive than the single options. Participants were given two concurrent decisions in which they were requested to choose between buying pairs of common consumer products that either were means-end related or not related to each other. When the participants were forced to make their choices, they chose the pairs of means or ends consumer products presumably because of the additional benefits they gave to these products. A conclusion from this study was that the integration of concurrent outcomes would only take place under a forced condition.

Boe and Gärling [30] conducted two experiments in order to investigate the hypothesis that the LSP could explain if the outcomes of two concurrent risky decisions would be integrated. As proposed by the LSP, only expected loss outcomes of concurrent decisions would then be integrated. The results yielded by the first experiment (Experiment 1) gave support to the LSP in that a prior outcome was integrated with the expected loss outcome of a current decision. As the expected loss outcomes of two concurrent decisions was not integrated, no integration was found regarding concurrent decisions. A possible proposed explanation that outcomes becomes ignored if they have not yet occurred was investigated in a second experiment (Experiment 2). Here the participants were offered bonuses with the thinking that this would increase their sensitivity they would have towards either to gains or losses. Again, no integration of the outcomes of concurrent decisions was yielded, although there was an effect of the bonus. Looking at the results, a question was raised as to the LSP could be extended to explain any integration of prior outcomes when choices were made between losses or between gains. A possible explanation of the lack of integration of could be that expectations of future risky outcomes simply becomes ignored when a DM is about to make decisions [31].

\section{Purpose of the Study}

The aim of the present experiment was to investigate if the losssensitivity hypothesis is capable of also explaining how concurrent decisions are integrated. It then needs to be assumed that one of the decisions are superordinate to the other. In the experiment, this was operationalized by imposing an order on the choices. Thus, when making the second choice it was hypothesized that subjects would take into account the outcome of the first choice. However, according 
to the hypothesis subjects would do that only if the outcome of the second choice was a potential loss. Therefore, the second choice was either between a sure loss and an uncertain loss or a sure gain and an uncertain gain. Integration was expected in the first but not in the latter case.

There are two possible reasons why a prior decision is not integrated with a current decision. The first reason is that there are too many outcomes of the first decision to take into account. This possibility was partly eliminated in the experiment since each of two options in the first decision only had two possible outcomes which either were both gains or nothing or both losses or nothing. In addition, if an order is imposed on a pair of concurrent decisions, it seems reasonable to assume that the DM only considers the outcomes of the chosen option. By setting up the first decision problem so that the expected value was much higher for one option and therefore very likely to be chosen, it was possible to test the assumption that subjects only take into account the outcomes of the chosen option.

The other reason for not integrating two concurrent decisions is that the outcome of the first decision is uncertain $[23,24]$. However, somewhat paradoxically, Kahneman and Tversky [15] report that subjects in a two-stage gamble ignored that reaching the second stage was uncertain. Why then do subjects not ignore the uncertainty of a prior outcome?

In three between-subjects conditions different subjects were given pairs of concurrent decisions, decisions with prior outcomes, and twostage gambles, respectively. The prior outcomes were either gains or losses which corresponded exactly to the outcomes of the dominant options of the first decisions in the condition with concurrent decisions, whereas in the two-stage gambles the probability of reaching the second stage was the same as obtaining the outcome of the dominant options of the first decisions in the condition with concurrent decisions. The second decisions were the same in all conditions. All subjects were also given a second block with only the second decisions. Thus, the existence of integration effects was possible to assess in withinsubjects comparisons in each of the between-subjects conditions. Based on prospect theory it was predicted that subjects in the second decisions would chose the sure gain but the uncertain loss. However, if integrating a prior gain, subjects would when facing a sure and an uncertain loss chose the former because it would be framed as a sure gain. If integrating a prior loss, subjects would be even more inclined to choose the uncertain loss. No integration was predicted for second decisions entailing only gains.

Under the conditions of the experiment (imposed order, a small number of possible outcomes of the prior decision) the same integration results were predicted in the condition with concurrent decisions. Consistent with the findings of Kahneman and Tversky [15] subjects were expected to ignore the uncertainty of the first stage in the two-stage gambles, thus leading to no integration in this condition. This result would also be consistent with integration in the condition with concurrent decisions. Boe [32] for an overview of theories and research results related to simultaneous decision-making).

\section{Materials and Methods}

\section{Subjects}

Forty-eight undergraduates participated as subjects. They were recruited from an available pool of subjects who on prior occasions had indicated that they were willing to participate. An equal number of men and women were randomly assigned to three conditions. Subjects were either paid the equivalent of $\$ 7$ or were offered three lottery tickets worth approximately the same amount.

\section{Design}

The design was mixed factorial with type of decision problem (concurrent decisions, decisions with prior outcome, and two-stage gambles) as a between-subjects factor. Within-subject factors were whether the first stage was included or not, whether the outcomes of the second decision were gains or losses, and, in the between-subjects conditions with concurrent decisions and with prior outcomes, whether the outcomes of the first decisions (or the prior outcomes) were gains or losses. In the stage-gamble condition, the latter was treated as a dummy factor. Different amounts of gains/losses were nested under the other conditions.

\section{Materials}

The materials consisted of fictitious bets which were presented on a computer screen. In all conditions, there was one block of single decisions, half of them consisting of a choice between an even chance of winning a certain amount or nothing and winning half the amount for sure. The other half consisted of an even chance of losing a certain amount or nothing and losing half the amount for sure. For different bets the amounts were SEK 80 and 40 (the equivalent of $\$ 11$ and 5.5), SEK 160 and 80 (the equivalent of $\$ 22$ and 11), SEK 240 and 120 (the equivalent of $\$ 33$ and 16.5), and SEK 320 and 160 (the equivalent of $\$ 44$ and 22). Each of the bets were presented twice.

A second block differed between the different between-subjects conditions. In the concurrent-decisions condition, subjects were asked to consider two choices, which were presented at the same time as follows. In the first choice, subjects had in half the cases an equal chance of either winning SEK 160 or 120,320 or 240,480 or 360 , or 640 or 480 .

Imagine that you will play the following two games. First consider both games. Indicate then your chosen alternative for each game.

1 You have $50 \%$ chance to lose/win $\mathrm{x}$ kronor, or

2 You have $50 \%$ chance to lose/win y kronor.

3 You lose/win $\mathrm{x}$ kronor for sure, or

4 You have $50 \%$ chance to lose/win y kronor, or $50 \%$ chance of losing/ winning nothing.

In the other half of the cases, subjects had an equal chance of losing SEK 200 or 160,400 or 320,600 or 480 , or 800 or 640 . Thus, one option always dominated the other one. The second choice was the same as in the block of single decisions. Each one was combined once with gains in the first choice, once with losses.

In the prior-outcome condition, subjects were given the following choices:

Imagine that you will play two games.

In the first game, you won/lost $\mathrm{x}$ kronor. Which of the following alternatives would you chose in the second game?

1 You lose/win $\mathrm{x}$ kronor for sure, or

2 You have $50 \%$ chance to lose/win y kronor or $50 \%$ chance to lose/ win nothing.

The prior gains or losses were the same as the dominant options in the first choice of the concurrent-decisions condition. In other respects, everything was the same. 
In the stage-gamble condition, the bets were as follows:

Imagine that you play the following two games. In the first game, you have $50 \%$ chance to continue to the second game so that you will be able to choose between the following alternatives.

1 You lose/win $\mathrm{x}$ kronor for sure, or

2 You have $50 \%$ chance to lose/win y kronor or $50 \%$ chance to lose/ win nothing.

Everything else was the same as in the other conditions. Each bet was presented twice.

\section{Procedure}

Subjects served in groups of four or less. When coming to the laboratory, they were given general instructions about how to perform the experiment. Subjects were also given the information that their participation was voluntary and that they could withdraw from the experiment at any time. In all three types of decision problems (concurrent decisions, decisions with prior outcome, and two-stage gambles), the block with single decisions were for half of the subjects presented first, for the other half last. An unrelated study and recall task was performed for five minutes in between. On each trial subjects first typed in the number of the chosen option, then subjects indicated on a scale from 1 to 100 how much they preferred it. This number was typed in after the choices. In the concurrent-decisions condition, subjects were first asked to make the first choice followed by the ratings, then the second choice followed by the ratings.

The sessions lasted for approximately 25 minutes. All subjects were then debriefed and paid.

\section{Results}

If subjects chose the certain option (or the dominant option), the ratings were assigned a positive value whereas they were otherwise assigned a negative value. Three missing values were replaced with the mean of the other subjects in the same condition.

Table 1 shows the mean ratings and the percentages of choices of the dominant option in the first decision in the concurrent-decisions condition. As may be seen, the dominant options were chosen very frequently. A 2 (current decision: gain vs. loss) by 2 (subsequent decision: gain vs. loss) analysis of variance (ANOVA) did not yield any significant effects of current choice at $\mathrm{p}=0.05$.

Table 2 gives the mean ratings and the percentages of choices of the certain option in the second decisions in the concurrent-decisions, prior-outcome, and stage-gamble conditions (prior loss or prior gain), and in the corresponding single decisions (no prior outcome).

It is suggested that no integration occurred since there were small differences between the single decisions and the other conditions. However, as expected, subjects were risk seeking when choosing between losses and risk aversive when choosing between gains. A 3 (condition: concurrent decisions vs. decisions with prior outcomes vs. two-stage gambles) by 2 (prior outcome: no prior outcome vs. prior loss vs. prior gain) by 2 (current decision: gain vs. loss) mixed ANOVA with repeated measures on the last two factors yielded a significant main effect of current decision, $\mathrm{F}(1,45)=4,72, \mathrm{p}<0.05$, Mse $=3009.3$, and of prior outcome, $\mathrm{F}(2,90)=5,58, \mathrm{p}<0.01, \mathrm{MSe}=1230.3$. Current decision also reliably interacted with prior outcome, $F(2,90)=3,53, p=0.05$, $\mathrm{MSe}=2878$.0. A significant interaction condition by prior outcome was also obtained, $\mathrm{F}(4,90)=3.80, \mathrm{p}<0.01, \mathrm{MSe}=4678,8$.

Separate ANOVAs for each condition suggested that the effect of prior outcome and the interaction between current decision and prior outcome was only reliable in the concurrent-decisions condition. Additional ANOVAs showed that prior outcome had a significant effect on current choices between losses but not on current choices between gains. After a prior loss, subjects became more risk aversive and were more willing to choose the certain alternative.

\section{Conclusion}

The present study aimed at investigating whether the losssensitivity hypothesis is capable of accounting for how concurrent decisions are integrated. It was then assumed that one of the decisions were superordinate to the other. However, the present results failed to support the hypothesis, as no integration occurred in the conditions with concurrent decisions and prior outcomes.

The loss-sensitivity hypothesis implies that subjects integrate losses with a prior outcome when evaluating the outcomes of a current choice. The separate ANOVAS showed that there were significant effects of a prior outcome on choices between losses but not on current gains, which is in accordance with the hypothesis.

One reason for not integrating two concurrent decisions is that the outcome of the first decision is uncertain $[23,24]$.

However, despite uncertainty, in the present experiment prior outcome had an effect in the concurrent-decisions whereas no effect was observed in the prior-outcome condition where the prior outcome

\begin{tabular}{|c|c|c|c|c|c|c|c|}
\hline \multicolumn{4}{|c|}{ Current choice/loses } & \multicolumn{4}{c|}{ Current choice/gains } \\
\hline Subsequent choice/Losses & Subsequent & \multicolumn{2}{c|}{ Shoice/Gains } & \multicolumn{2}{c|}{ Subsequent Choice/Losses } & Subsequent choice/Gains \\
\hline M & $\%$ & M & $\%$ & M & M & \\
\hline 56.4 & 87.5 & 49.8 & 87.5 & 54.0 & 84.4 & 63.3 \\
\hline
\end{tabular}

Table 1: Mean ratings and percentages of choices of dominant option in the first decision in concurrent-decisions conditions.

\begin{tabular}{|c|c|c|c|c|c|c|c|c|c|c|c|c|}
\hline \multirow{3}{*}{$\begin{array}{c}\text { Current choice } \\
\text { Condition }\end{array}$} & \multicolumn{4}{|c|}{ No prior outcome } & \multicolumn{4}{|c|}{ Prior loss } & \multicolumn{4}{|c|}{ Prior gain } \\
\hline & \multicolumn{2}{|c|}{ Losses } & \multicolumn{2}{|c|}{ Gains } & \multicolumn{2}{|c|}{ Losses } & \multicolumn{2}{|c|}{ Gains } & \multicolumn{2}{|c|}{ Losses } & \multicolumn{2}{|c|}{ Gains } \\
\hline & $M$ & $\%$ & $M$ & $\%$ & $M$ & $\%$ & $M$ & $\%$ & $M$ & $\%$ & M & $\%$ \\
\hline Stage-gamble ${ }^{1}$ & -14.6 & 36.7 & -8.5 & 41.4 & -16.7 & 45.3 & -8.5 & 35.9 & 26.0 & 26.6 & -3.5 & 37.5 \\
\hline Prior-outcome & -29.7 & 22.7 & 7.3 & 51.5 & -16.6 & 45.3 & 5.0 & 31.3 & -24.9 & 40.6 & -5.1 & 39.0 \\
\hline $\begin{array}{l}\text { Concurrent- } \\
\text { decisions }\end{array}$ & -25.5 & 32.8 & 29.1 & 71.1 & 18.9 & 50.0 & 22.4 & 46.9 & -28.2 & 51.6 & 18.5 & 42.2 \\
\hline
\end{tabular}

Table 2: Mean ratings and percentages of choices of certain option in the different conditions. 
Citation: Boe O, Garling T (2018) Using the Loss-Sensitivity Principle to Explain the Integration of Concurrent Decisions in Computer-based Fictitious Betting Situations. Arts Social Sci J 9: 423. doi: 10.4172/2151-6200.1000423

was known. In the stage-gamble condition subjects seemed to ignore the uncertainty. The effect of prior outcome in the concurrent-decisions condition may not reflect integration.

It is possible that integration will not occur unless some other conditions are prevalent. One intriguing possibility to obtain integration may be to increase saliency of the loss outcomes of the current choice, for instance, by increasing the amount or to threat subjects with a fine. Increasing positive mood is another possibility $[33,34]$.

\section{Acknowledgements}

This research was financially supported by grant \#F77/95 to Professor Tommy Gäling at the Department of Psychology at Gothenburg University from the Swedish Council Research in the Humanities and Social Sciences. This research was also sponsored by the University of South-Eastern Norway. The authors wishes to thank senior lecturer Merete Ruud at the Norwegian Military Academy and Pål Johan Karlsen, editor of Scandinavian Psychologist, for valuable help with the language of this article as well as valuable comments.

\section{References}

1. Brehmer B (1992) Dynamic decision making: Human control of complex systems. Acta Psychol 81: 211-241.

2. Huber $O$ (1990) Cognitive processes in a multistage investment task. In: Gilhooly KJ, Keane M, Logia RH, Erdos G (eds.) Lines of thinking: Reflections on the psychology of thought (Vol. 1). Wiley, Chitchester, UK 327-336.

3. Boutilier C, Dean T, Hanks S (1999) Decision-theoretic planning: Structural assumptions and computational leverage. J Artificial Intelligence Res 11: 1-94.

4. Boutilier C, Dearden R (1996) Approximating value trees in structured dynamic programming. Proceedings of the 13th International Conference on Machine Learning 54-62.

5. Boutilier C, Goldszmidt M (1995) Exploiting structure in policy construction. Proceedings of the 14th International Joint Conference on Artificial Intelligence 1104-1111.

6. Dean T, Givan R, Kim K (1998) Solving planning problems with large state and action spaces. Proceedings of the 4th International Conference on Artificial Intelligence Planning Systems (ICAPS-98) 102-110.

7. Guestrin C (2003) Planning Under Uncertainty in Complex Structured Environments. Stanford University, Stanford, CA, USA.

8. Marthi B, Russell S, Latham D, Guestrin C (2005) Concurrent hierarchical reinforcement learning. Adaptive Behavior 13: 165-188.

9. Mausam M, Weld DS (2004) Solving concurrent Markov decision processes Proceedings of the 19th National Conference on Artificial Intelligence (AAAI 04) 716-722.

10. Mausam, M, Weld DS (2005) Concurrent probabilistic temporal planning Proceedings of the 15 th International Conference on Planning and Scheduling 1-10.

11. Russell S, Zimdars AL (2003) Q-decomposition for reinforcement learning agents. University of California, Berkeley.

12. Singh S, Cohn D (1998) How to dynamically merge Markov decision processes. Neural Information Processing Systems 10: 1057-1063.
13. Younes $H$, Simmons $R$ (2004) A formalism for stochastic decision processes with asynchronous events. AAAl Workshop on Learning and Planning in Markov Processes: Advances and Challenges.

14. Precup D (2000) Temporal Abstraction in Reinforcement Learning. Amhearst: University of Massachusetts, Amherst.

15. Kahneman D, Tversky A (1979) Prospect theory: An analysis of decision under risk. Ekonometrika 47: 263-291.

16. Tversky A, Kahneman D (1991) Loss aversion in riskless choice: A referencedependent model. Quarterly J Economics 106: 1039-1061.

17. Tversky A, Kahneman D (1992) Advances in prospect theory: Cumulative representation of uncertainty. J Risk Uncertainty 5: 297-323.

18. Shafir E, Simonson I, Tversky A (1993) Reason-based choices. Cognition 49 $11-36$.

19. Neumann J, Morgenstern O (1947) Theory of games and economic behavior Wiley, New York.

20. Kahneman D, Tversky A (1983) Choices, values, and frames. American Psychologist 39: 341-350.

21. Tversky A, Kahneman $D$ (1981) The framing of decisions and the psychology of choice. Sci 211: 453-458.

22. Boe O, GArling T (2015) Effects of causal relatedness and uncertainty on integration of outcomes of concurrent decisions. Procedia-Social Behaviora Sciences 190: 113-119.

23. Garling T, Romanus J (2008) Integration and segregation of prior outcomes in risky decisions. Scandinavian J Psychology 38: 289-296.

24. Tversky A, Shafir E (1992) The disjunction effect in choice under uncertainty Psychological Sci 3: 305-309.

25. Rohanimanesh K (2006) Concurrent decision making in Markov decision processes. University of Massachusetts Amherts, Boston.

26. Thaler RH, Johnson EJ (1990) Gambling with the house money and trying to break even. The effects of prior outcomes on risky choice. Management Sci 36: 643-660.

27. Linville PW, Fischer GW (1991) Preferences for separating or combining events. J Pers Soc Psychol 60: 5-23.

28. Boe O (2015) A further investigation of the attentional bias in concurrent decisions. Procedia-Social Behavioral Sciences 190: 377-383.

29. Boe O, Gärling T (2015) Failures to integrate causally related outcomes of concurrent decisions. Procedia-Social Behavioral Sciences 190: 287-292.

30. Boe O, Gärling T (2015) Loss sensitivity and integration of outcomes of concurrent risky decisions. Procedia-Social Behavioral Sciences 190: 550-558.

31. Lovallo D, Kahneman D (2003) Delusions of success: How optimism undermines executives' decisions. Harv Bus Rev 81: 56-63.

32. Boe O (2015b) Simultaneous decision-making: An overview of theories and research results. J Progessive Res Social Sciences 3: 114-132.

33. Isen A (1987) Positive affect, cognitive processes, and social behavior Advances in Experimental Social Psychology 20: 203-253.

34. Romanus J, Hassing L, Gärling T (1996) A loss-sensitivity explanation of integration of prior outcomes in risky decisions. Acta Psychologica 93: 173-183. 